3. J. W. Milnor, On the cobordism ring $\Omega^{*}$, and a complex analogue. I, Amer. J. Math. 82 (1960), 505-521.

4. S. P. Novikov, Homotopy properties of Thom complexes, Mat. Sb. (N.S.) 57 (99) (1962), 407-442. (Russian)

5. R. Thom, Quelques proprietés globales des variêtés différentiable, Comment. Math. Helv. 28 (1954), 17-86.

6. G. W. Whitehead, Generalized homology theories, Trans. Amer. Math. Soc. 102 (1962), 227-283.

INSTITUTE FOR ADVANCED STUDY AND UNIVERSITY OF VIRGINIA

\title{
A NOTE ON APPROXIMATION BY BERNSTEIN POLYNOMIALS
}

BY B. BAJŠANSKI AND R. BOJANIĆ

Communicated by A. Zygmund, May 11, 1964

Let $f$ be continuous on $[0,1]$ and $0 \leqq \alpha<\beta \leqq 1$ and let $B_{n} f$ be the Bernstein polynomial of $f$ of degree $n$, defined by

$$
B_{n} f(x)=\sum_{\nu=0}^{n} f\left(\frac{\nu}{n}\right)\left(\begin{array}{l}
n \\
\nu
\end{array}\right) x^{\nu}(1-x)^{n-\nu} .
$$

In view of a result of $\mathrm{E}$. $\mathrm{V}$. Voronovskaya, which states that the boundedness of $f$ on $[0,1]$ and the existence of $f^{\prime \prime}$ at a point $x \in[0,1]$ implies that

$$
B_{n} f(x)-f(x)=\frac{x(1-x)}{2 n} f^{\prime \prime}(x)+o\left(\frac{1}{n}\right) \quad(n \rightarrow \infty),
$$

it has been conjectured $[1$, p. 22] that the relation

$$
B_{n} f(x)-f(x)=o\left(\frac{1}{n}\right)
$$

cannot be true for all $x \in[\alpha, \beta]$ unless $f$ is a linear function on $[\alpha, \beta]$. The following theorem related to this conjecture was proved by K. de Leeuw [2]:

If $f$ is continuous on $[0,1]$ and

$$
B_{n} f(x)-f(x)=O\left(\frac{1}{n}\right)
$$


holds uniformly on every subinterval $[\alpha, \beta]$ of $[0,1]$ and if in addition

$$
B_{n} f(x)-f(x)=o\left(\frac{1}{n}\right)
$$

at almost all points of $[\alpha, \beta]$, then $f$ is linear on $[\alpha, \beta]$.

We shall give here a simple proof of the original conjecture.

THEOREM. If $f$ is continuous on $[0,1]$ and

$$
B_{n} f(x)-f(x)=o\left(\frac{1}{n}\right) \quad(n \rightarrow \infty)
$$

holds for each fixed $x \in(\alpha, \beta)$, then $f$ is a linear function on $[\alpha, \beta]$.

Proof. To make the argument as transparent as possible we list first the properties of Bernstein polynomials used in this proof.

(i) $B_{n}(f+g)=B_{n} f+B_{n} g$ and if $f \leqq g$ on $[0,1]$ then $B_{n} f \leqq B_{n} g$.

(ii) If $h$ is a linear function on $[0,1]$, then $B_{n} h=h$.

(iii) If $Q(x)=A x^{2}+B x+C$, then $B_{n} Q(x)-Q(x)=A(x(1-x) / n)$.

(iv) If $g$ is bounded on $[0,1]$ and $g=0$ on $[\alpha, \beta]$, then $B_{n} g(x)$ $=o(1 / n)(n \rightarrow \infty)$ for each fixed $x$ in the interior of $[\alpha, \beta]$. (Actually, $B_{n} f(x)=O\left(e^{-\delta(x) n}\right) \quad(n \rightarrow \infty)$, with $\delta(x)>0$, but the weaker property is sufficient.)

We need also the following lemma:

LEMma. If $f$ is continuous on $[\alpha, \beta]$, vanishes at $\alpha$ and $\beta$ and has a positive maximum on $[\alpha, \beta]$ then there is a quadratic polynomial $Q(x)$ $=A x^{2}+B x+C$ with $A<0$ such that

$$
f(x) \leqq Q(x) \text { for all } x \in[\alpha, \beta]
$$

and

$$
f(c)=Q(c) \text { for some } c \text { in the interior of }[\alpha, \beta] .
$$

This lemma is geometrically almost obvious. We can namely choose the parabola $P(x)=A x^{2}+B x+C^{*}$ with $A<0$ such that its arc over $[\alpha, \beta]$ lies in the strip $M \leqq y \leqq 3 M / 2$ where $M=\max _{\alpha \leq x \leq \beta} f(x)>0$. If $d=\min _{\alpha \leq x \leq \beta}(P(x)-f(x))$, then the quadratic polynomial $Q(x)$ $=P(x)-d$ has the required properties.

To prove the theorem, suppose that $f$ satisfies (1) for each $x \in[\alpha, \beta]$. By subtracting a suitable linear function and using (ii), if necessary, we may assume that $f(\alpha)=f(\beta)=0$. We have to show that $f=0$ on $[\alpha, \beta]$.

Assume that the maximum of $f$ on $[\alpha, \beta]$ is positive. Then by the 
preceding lemma we can find a polynomial $Q$ satisfying (2) and (3). Since by (2) $f(x) \leqq Q(x), x \in[\alpha, \beta]$, we can find a bounded function $g$ on $[0,1]$ such that $g=0$ on $[\alpha, \beta]$ and

$$
f(x) \leqq Q(x)+g(x) \text { for all } x \in[0,1] .
$$

Applying (i) we get

$$
B_{n} f(x) \leqq B_{n} Q(x)+B_{n} g(x) .
$$

Putting here $x=c$ and using (3) we get

$$
B_{n} f(c)-f(c) \leqq B_{n} Q(c)-Q(c)+B_{n} g(c) .
$$

Since $c$ is in the interior of $[\alpha, \beta]$ we have by (iv) $B_{n} g(c)=o(1 / n)$ $(n \rightarrow \infty)$. Using this result and (iii) we obtain

$$
B_{n} f(c)-f(c) \leqq A \frac{c(1-c)}{n}+o\left(\frac{1}{n}\right) \quad(n \rightarrow \infty),
$$

with $A<0$, which is impossible by (1). Thus the maximum of $f$ on $[\alpha, \beta]$ cannot be positive.

Likewise, by considering $-f$ instead of $f$, we see that the minimum of $f$ on $[\alpha, \beta]$ cannot be negative.

Thus, $f=0$ on $[\alpha, \beta]$, and the theorem is proved.

\section{REFERENCES}

1. G. G. Lorentz, Bernstein polynomials, University of Toronto Press, Toronto, 1953.

2. $\mathrm{K}$. de Leeuw, On the degree of approximation by Bernstein polynomials, J. Analyse Math. 7 (1959), 89-104.

Ohio State University 